\title{
Perspective
}

PERSPECTIVE Actualité en histoire de l'art

$2 \mid 2021$

Habiter

\section{Une architecture performative}

Un débat entre Mathilde Chénin, Christopher Dell, Matthieu Duperrex, Marion Howa et Fanny Léglise, modéré par Tiphaine Abenia et Daniel Estevez

Tiphaine Abenia, Mathilde Chénin, Christopher Dell, Matthieu Duperrex, Daniel Estevez, Marion Howa et Fanny Léglise

\section{(2) OpenEdition}

Journals

Édition électronique

URL : https://journals.openedition.org/perspective/24955

DOI : $10.4000 /$ perspective. 24955

ISSN : 2269-7721

Éditeur

Institut national d'histoire de l'art

Édition imprimée

Date de publication : 30 décembre 2021

Pagination : 67-86

ISBN : 978-2-917902-92-9

ISSN : $1777-7852$

Ce document vous est offert par Université Toulouse 2 - Jean Jaurès

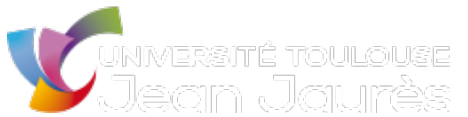

Référence électronique

Tiphaine Abenia, Mathilde Chénin, Christopher Dell, Matthieu Duperrex, Daniel Estevez, Marion Howa et Fanny Léglise, «Une architecture performative », Perspective [En ligne], 2 | 2021, mis en ligne le 30 juin 2022, consulté le 21 février 2022. URL : http://journals.openedition.org/perspective/24955 ; DOI https://doi.org/10.4000/perspective.24955 


\title{
Une architecture performative
}

\author{
Un débat entre Mathilde Chénin, Christopher Dell, \\ Matthieu Duperrex, Marion Howa et Fanny Léglise, \\ modéré par Tiphaine Abenia et Daniel Estevez
}

Si vous vivez la mondialité, vous êtes au point de combattre

vraiment la mondialisation ${ }^{1}$.

À l'issue de la présentation du pavillon français de la XVII ${ }^{\mathrm{e}}$ Biennale d'architecture de Venise, l'équipe lauréate, sous l'égide de Christophe Hutin Architecture, a voulu engager une réflexion croisée sur le concept d'habiter en architecture. Cette notion est abordée dans l'exposition sous l'angle de la " mondialité vécue ", selon l'expression du poète Édouard Glissant, une plongée dans des archipels de lieux, de moments et d'expériences architecturales. La proposition portée par le pavillon s'intitule " Les communautés à l'œuvre ${ }^{2} "$. Elle tente de formuler une réponse directe à la question posée aux participants internationaux par le commissaire général de la Biennale Hashim A. Sarkis : "Comment vivrons-nous ensemble ? ", qui prend pour point de départ l'habiter et l'action habitante dans la production de la ville contemporaine : observons nos façons de vivre ensemble aujourd'hui, partout dans le monde, pour tenter de tracer un avenir en commun possible et souhaitable à nos villes. Partons donc de ce qui existe déjà sous nos yeux. Posons-nous la question suivante : l'action ordinaire des communautés habitantes sur leur environnement peut-elle être considérée à la même hauteur que l'action savante des architectes, ingénieurs et urbanistes qui conçoivent les villes ?

La revue Perspective a permis de lancer le débat en sollicitant différents regards sur les problématiques posées par l'exposition du Pavillon français et développées dans son catalogue. Les contributeurs et contributrices à ce débat sont en effet issus de différents horizons, mais abordent dans leurs travaux de nombreux éléments théoriques soutenus dans l'exposition internationale de Venise. En effectuant des détours par l'art, l'architecture et l'urbanisme, ce débat tente donc de questionner, de manière plurielle, nos conceptions de l'habiter au-delà de ses formes les plus évidentes, ainsi que le rôle des communautés habitantes dans ces différentes manières de construire et d'habiter le monde.

Point pivot des discussions organisées ici, l'exposition proposée par le Pavillon français à Venise donne à voir certaines puissances productives de l'architecture habitée, autrement dit certaines capacités et potentiels des édifices qui trouvent leur origine dans les actes concrets de l'habiter. Elle décrit ainsi différentes expérimentations de terrain menées par l'équipe sur le thème de la performance habitante. La scénographie mobilisée 
pour mettre en contraste ce propos consiste en un agencement spatial de documents vidéographiques portant sur ces opérations de terrain, en multi-projection. Il s'agit d'offrir une expérience perceptive à partir des déplacements, des choix et des hasards qui s'offrent à chaque visiteur dans l'espace architectural du pavillon. Assemblage non narratif de films considérés comme autant d'échantillons, le dispositif d'exposition vise donc à activer la perception des spectateurs. À l'instar des travaux eux-mêmes, leur présentation en multi-projection vidéo s'inscrit dans le thème de la performativité de l'architecture.

De ce point de vue, l'une des hypothèses théoriques fortes de l'exposition concerne la relation étroite entre structure et design architectural. Nous entendons par structure une condition collective, à la fois matérielle et immatérielle, soutenant l'expression d'individualités multiples au sein d'un cadre partagé. Une structure efficiente libère et augmente les capacités d'action des habitants sur leurs milieux de vie, une structure impensée tend à les limiter. Dès lors, l'hypothèse posée affirme ainsi qu'une conception architecturale fondée sur l'action habitante peut relever d'une dynamique conjointe entre compétence des structures d'une part et performance des habitants de l'autre. Ce schéma emprunte au champ linguistique qui, depuis Ferdinand de Saussure, a analysé la performativité linguistique à partir de la distinction fondamentale entre la langue, comme savoir formel, et la parole, comme traduction effective de ce savoir.

Le débat rapporté dans le texte qui suit tente lui aussi d'illustrer la performativité que peut recéler tout échange collectif, considéré comme une dynamique de confrontations et de relations, une navigation à la fois libre et structurée. C'est pourquoi il conviendrait mieux de parler ici de conversation. On rappellera à cet égard la formule du chercheur et musicien Donald A. Schön qui, dans son livre Le Praticien réflexif, définissait déjà la conception architecturale comme une " conversation réflexive avec la situation ${ }^{3}$ ", que l'on peut rapprocher, on le verra plus loin, des démarches d'improvisation basées sur la structure.

Notre conversation est accompagnée d'une série photographique dont les images proviennent directement des travaux et expérimentations présentés dans le Pavillon français. Cette série respecte une structure dialogique : des couples d'images successifs entrent ainsi en résonance entre elles et avec les sujets abordés dans la discussion. Pourquoi recourir de façon si fréquente à la fragmentation des informations ? L'organisation des images, dans le présent texte tout comme dans l'exposition de Venise ou encore dans le contenu de son catalogue, cherche à installer un " dissensus représentationnel ". Garantie d'une perception active, le principe de dissensus est aussi ce qui fonde, si l'on en croit Jacques Rancière, tout procédé de représentation critique. On connaît la définition qu'en propose le philosophe : " En son sens originel, critique veut dire : qui concerne la séparation, la discrimination. Critique est l'art qui déplace les lignes de séparation, qui met de la séparation dans le tissu consensuel du réel, et, pour cela même, brouille les lignes de séparation qui configurent le champ consensuel du donné ${ }^{4}$."

Dualité, trialectique, multiplicité, diagrammatique, sérialité... toutes les opérations de fragmentation du perçu correspondent à des principes qui semblent traverser les procédures de conception critique en architecture. D'ailleurs, le traditionnel géométral d'architecte, plan-coupe-élévation, n'est-il pas déjà lui-même une procédure de fragmentation ? Nous envisageons, dès lors, la conception architecturale dans sa dynamique critique, dans sa capacité de redescription et de réorganisation des situations - une posture de conception qui s'appuie à nouveau sur les propos de Rancière : " toute situation est susceptible d'être fendue en son intérieur, reconfigurée sous un autre régime de perception et de signification ${ }^{5}$ ".

[Tiphaine Abenia et Daniel Estevez] 


\section{Habiter, résister}

- Tiphaine Abenia et Daniel Estevez. Le Pavillon français de la XVII Biennale d'architecture de Venise défend l'idée que les communautés contemporaines réinventent aujourd'hui leurs significations et leurs pratiques habitantes à partir d'actions précises situées en des lieux précis ${ }^{6}$. On y souligne notamment que les communautés naissent aussi à partir de situations de dissensus. Voici que des groupes d'habitants-résistants augmentent, par leurs actions même, leur structure sociale, leurs savoirs environnementaux, leur connaissance du patrimoine spatial, leurs compétences juridiques et jusqu'à leur propre pouvoir politique. Des recherches en sciences sociales identifient ce phénomène par l'expression « résistance éclairée $^{7}$ » qui définit également une manière $d^{\prime}$ habiter pleinement et intensément son territoire de vie. Pour agir ici et maintenant, il semble donc utile de s'émanciper des seuls savoirs institutionnalisés et de développer parallèlement des expertises populaires et spécifiques aux habitats concernés. Dans ces conditions, peut-on envisager une conception architecturale dans laquelle agir communautaire et dissensus constituent un objectif prioritaire? Selon votre expérience, une réappropriation collective de l'acte d'habiter intégrant des formes de conflictualités vous apparaîtelle observable?

- Mathilde Chénin. Les communautés qui naissent depuis une situation de dissensus me semblent intéressantes, non parce qu'elles se constituent en rapport à un adversaire commun, mais bien parce qu'elles émergent, comme vous l'évoquez, en relation à un lieu, à un milieu particulier. La sociologue Laura Centemeri montre cela précisément dans un article qui retrace quarante années de luttes locales contre l'extension de l'aéroport de Milan-Malpensa : ce qui va constituer le ciment de cette communauté d'habitantsrésistants, c'est le rapport au lieu, l'expérience habitante justement qu'elle en fait. Cette expérience habitante, qui se noue en chaque personne au gré de tout un répertoire de gestes, de pratiques, de présences mais aussi d'émotions, devient le substrat d'une manière très singulière de faire commun. Singulière, car en faisant appel à des manières d'être au monde qui ont trait au familier, à l'aisance et à la proximité du chez-soi, cette relation s'adosse à un attachement, à une affection particulière pour le lieu. Centemeri nomme cette manière de se mettre en commun, le mode d'engagement " en résonance ${ }^{8}$ ». Cette expression est très belle et très juste, en ce qu'elle évoque la qualité vibratoire plus qu'identitaire de la relation au lieu que crée l'expérience habitante, celle d'une attention, d'une sollicitude, le désir de prendre soin. 1a-b. Délibérations dans les processus de projet.

[a] Réhabilitation du hameau de Beutre, à Mérignac, 2019.

[b] Au cinéma Le Sans Souci à Soweto (bidonville de Kliptown), atelier "Learning from » (ENSA Toulouse), 2014.
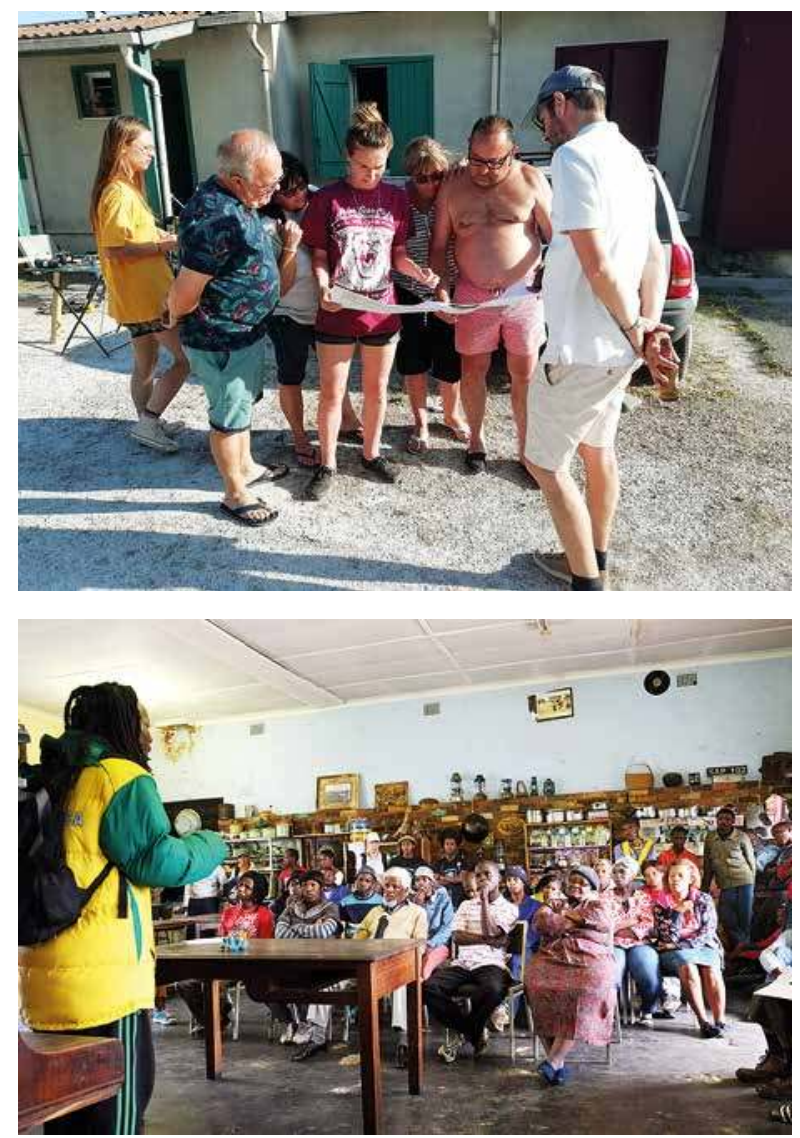
Accéder à la dimension relationnelle de l'habiter, c'est peut-être alors creuser à l'endroit de cet attachement, de cette relation de proche à proche, de cette connaissance mutuelle qui se tisse au fil d'un temps long. C'est peut-être d'abord et avant tout devenir ami d'un milieu donné ${ }^{\text {. }}$

- Tiphaine Abenia et Daniel Estevez. L'empathie avec son milieu de vie est certainement une condition de la vie en commun, mais ne pourrait-on pas aussi apparenter l'habiter au mésusage, l'appropriation à la transgression? N'est-ce pas alors la question de la désobéissance sous ses différentes formes qui se trouve posée dans une société humaine qui semble forcer au consensus (entendu ici au sens de Jacques Rancière, c'est-à-dire comme univocité d'interprétation du donné)?

- Marion Howa. Si je me réfère à ma propre expérience d'architecte, je parlerai de dissidence plutôt que de désobéissance. Examinons par exemple un cas d'étude présenté dans le Pavillon français et sur lequel j'ai particulièrement travaillé. II s'agit de la cité de Beutre à Mérignac, près de Bordeaux, un ancien camp de transit pour immigrés qui n'a reçu aucune intervention du bailleur social depuis sa création. Pour affronter cet abandon institutionnel, les habitants de la cité n'ont pas eu d'autres choix que de s'organiser par eux-mêmes. Pour réemployer la formule de Jacques Rancière définissant l'émancipation, Beutre est un cas exceptionnel, dans le paysage du logement social en France, où l'architecture suit « une intelligence qui n'obéit qu'à elle-même ${ }^{10}$ ». Face au désengagement historique du bailleur, les quatre-vingt-treize maisons de la cité ont été transformées par leurs locataires depuis cinquante ans.

Ainsi à Beutre, nous connaissons un habitant, appelons-le Monsieur V., qui a compris qu'il devait attendre une année encore avant le démarrage du chantier du projet de rénovation que nous menons au cas par cas. Il sait aussi que s'il s'occupe lui-même des travaux, ces derniers seront plus adaptés à ses projets et certainement mieux finis que s'ils sont le fait d'entreprises en marché public. Il sait enfin que le bailleur lui aurait interdit d'agrandir sa maison s'il en avait demandé l'autorisation : il est donc resté discret mais a engagé lui-même ses travaux. Comme l'institution est en défaut sur le maintien de son propre patrimoine depuis cinquante ans, Monsieur V. met les gestionnaires devant le fait accompli. Ironie de l'anecdote, ces derniers seront même admiratifs du transfert de responsabilité que ce locataire aura spontanément entrepris. Le bailleur dira : "L'autoconstruction, c'est l'avenir du logement social ! » Lucide, Monsieur V. connaît les endroits où il est efficace, sait comment négocier avec son bailleur et agir avec sa communauté. C'est dans ce sens que je dirais que Monsieur V. habite en dissident.

Dans le cas de cette cité de Beutre, la prise de pouvoir par une communauté sur I'architecture qu'elle habite est une forme de résistance à l'abandon du service social du logement. Croire que la responsabilité individuelle des habitants en logement locatif social est suffisante pour se substituer au service public d'un bailleur démissionnaire est toutefois illusoire ${ }^{11}$. Le pouvoir de Monsieur V. tient aux liens forts qui l'unissent à Beutre. Tous les bénéficiaires du logement social ne peuvent pas s'appuyer sur l'économie morale d'une communauté. À l'heure de l'affirmation du tournant néolibéral des politiques publiques françaises du logement, et dans un contexte de vulnérabilisation des personnes les plus précaires, compter sur la responsabilisation des habitants est tout simplement discriminant. Au lieu de voir dans l'agentivité ${ }^{12}$ - ou pouvoir d'agir - de Monsieur V. un modèle de "surhabitant » et d'attendre des autres bénéficiaires du logement social qu'ils aient la même énergie que lui, nous pouvons au moins réfléchir sur nous-mêmes. Nous, architectes, pouvons habiter la marge de notre métier. Faisons l'expérience de chercher - de se créer - les lieux $\mathrm{d}^{\prime}$ infiltration de notre pouvoir dans un système professionnel où rien n'est fait pour travailler de cette façon. Nous pourrons alors peut-être mettre en place des dispositifs ouverts à même 
de renforcer le pouvoir d'une communauté habitante dans un projet d'architecture ; mais nous ne pourrons pas, dans nos projets collaboratifs qui déploient une véritable écoute des habitants, assumer la disparition des indispensables services publics.

\section{Habiter en relation}

- Tiphaine Abenia et Daniel Estevez. Les carnets du poète chinois Li Yu ${ }^{13}$ (1611-1680 ?) nous font savoir que le phénomène de l'habiter peut être envisagé comme une constellation d'actes ordinaires que chacun peut accomplir. L'art de vivre que décrit Li Yu est performatif et foisonnant d'aventures personnelles minuscules, mais il fait monde. Ici, le quotidien s'oppose à l'idée de centre comme de périphérie, de forme unique comme d'espace monument ${ }^{14}$. $\mathrm{Ne}$ faudrait-il pas situer la réalité de l'architecture dans ce système des relations horizontales multiples entre les choses construites, les actes quotidiens et les personnes qui peuplent la ville ? L'architecte John C. Turner écrivait déjà, dans les années 1960 : «Les valeurs réelles sont celles qui résident dans les relations entre les éléments de l'action d'habiter, entre les habitants, leurs activités et leur environnement ${ }^{15}$. " Le poète Édouard Glissant, en faisant de la relation un concept clé de l'existence humaine, prolongeait aussi cette philosophie relationnelle : "La poétique de la Relation pressent, suppose, inaugure, rassemble, éparpille, continue et transforme ${ }^{16}$. » Aujourd'hui, nous voyons cette valeur de la relation s'étendre pour embrasser $d^{\prime} u n$ seul geste acteurs humains et non humains :

\footnotetext{
La seule analyse des relations entre les humains [...] ne suffit à rendre compte ni de la réalité des collectifs humains, ni de celle des individus humains en tant que ceux-ci se redéfinissent partiellement au travers de leurs multiples relations avec ces "non-humains" qui composent leurs divers milieux : animaux, molécules, objets techniques, divinités, procédures, matériaux, bâtiments ${ }^{17}$.
}

Habiter serait dès lors, avant tout, cohabiter, investir l'espace de la relation pour s'émanciper de l'espace marchandisé, de la vie prédatrice de ressources. Comment la conception architecturale contemporaine peut-elle accéder à cette réalité relationnelle de l'habiter ?

- Mathilde Chénin. La relation en soi n'est gage d'aucune émancipation. Peut-être était-il encore possible de croire le contraire entre la fin des années 1990 et le début du XXI siècle, quand un ensemble de pratiques et de démarches artistiques - ce que l'on a appelé l'esthétique relationnelle - a investi cette idée très vaste de la relation. Le programme était alors ambitieux. Les pratiques, les œuvres issues de cet art relationnel devaient permettre au champ de l'art contemporain de dessiner des « utopies de proximité ${ }^{18}$ » qui nous apprendraient à «mieux habiter le monde ». Nous assistions presque, par avance, à une " tentative d'atterrissage ${ }^{19}$ » de l'art et des artistes, concentrés qu'ils étaient à " construire des espaces concrets » ou encore à « inventer des relations possibles avec leurs voisins ${ }^{20}$ ». Nous en revenons depuis, les communautés créées par ces œuvres relevant bien souvent d'un entre-soi qui peine à adresser les questions primordiales et urgentes du vivre-ensemble. Pas tant parce qu'elles n'ouvrent un espace de convivialité qu'à un public restreint et bien particulier, mais plutôt parce qu'entendant l'idée même de communauté comme un tout harmonieux, univoque et réconcilié, ces œuvres et ces pratiques contribuent à nourrir la vision néolibérale du pluralisme démocratique comme cet espace supposément pacifié dans lequel il convient de s'assimiler plutôt que de chercher à se singulariser.

Vingt ans plus tard, nous sommes pétris d'injonctions à la relation, à une certaine forme de relation. L'esthétique relationnelle n'aura fait in fine qu'accompagner la digestion, la neutralisation de la relation par le puissant et implacable mouvement de commodification propre 

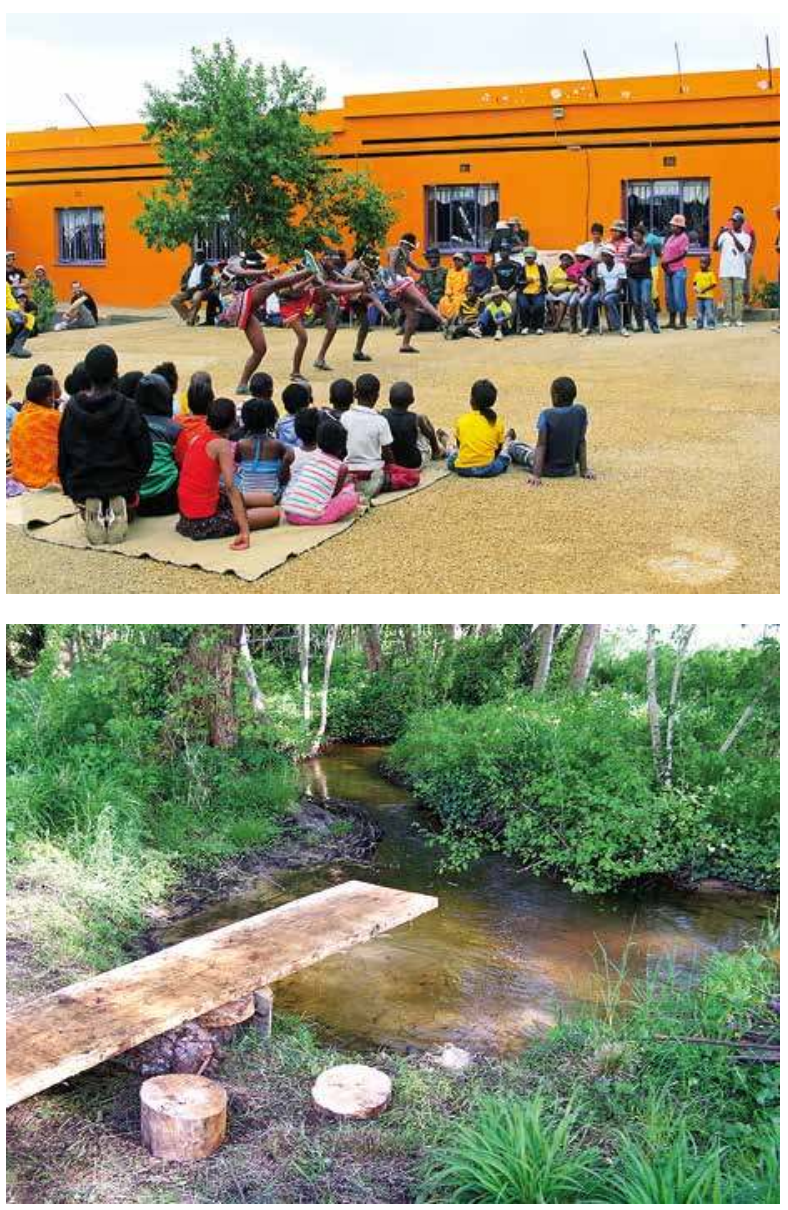

2a-b. "Puissance est relation ».

[a] Fête de fin de chantier à l'orphelinat Soweto Kliptown Youth à Soweto, atelier "Learning from » (ENSA Toulouse), 2012.

[b] Projet de ruisseau éducatif à Uzeste (Gironde), atelier « Learning from » (ENSA Toulouse), 2013. au néolibéralisme. Coworking, co-conception, co-construction, cocréation, coproduction, cohabitation : le suffixe « co- » est d'autant plus généralisé qu'il a du plomb dans l'aile, ne garantissant plus à lui seul la teneur des relations qu'il active. À l'image des tiers lieux par exemple, qui émergent joyeusement à l'orée des années 2000 depuis des hackerspaces empreints d'un souci égalitariste et libertaire ${ }^{21}$, et qui sont devenus en l'espace de vingt ans des avant-postes du néolibéralisme et de la promotion de l'entrepreneur libre et indépendant.

Autrement dit, vivre à côté de n'est pas vivre avec. Et pour répondre à votre question, avant même de se demander comment la conception architecturale peut " accéder à la réalité relationnelle de l'habiter", il me semble qu'il convient de s'interroger d'abord sur la qualité des relations, sur le comment des relations que l'on cherche à créer ${ }^{22}$. Et ce n'est pas chose aisée, alors que la puissance de la relation telle que la pensait Édouard Glissant a été en grande partie dé-substantialisée par ce mouvement de commodification que j'évoquais à l'instant - aussi et surtout dans un contexte qui voit les espaces sociaux se polariser drastiquement à force de tensions qui rendent presque impossible l'idée même d'une mise en commun. Difficile dans ce contexte de plaider en faveur d'espaces relationnels « antagonistes ${ }^{23}$ » ou pour une " coexistence activement conflictuelle ${ }^{24}$ ». Pourtant, accepter, faire place, se laisser affecter par des idées, des ressentis, des vécus, des formes autres - et potentiellement

antagonistes - semble être le seul chemin pour que la relation s'étoffe dans la rencontre, plutôt que de s'éteindre dans l'assimilation.

Ouvrir les collectifs humains à l'ensemble des entités qui les peuplent, non-humains et non-vivants, est certainement un premier pas sur ce chemin. Les termes non-humains, non-vivants me laissent pourtant mal à l'aise. Cette définition par la négative - les « non- »n'a rien de satisfaisant ${ }^{25}$. Elle est le symptôme lexical de notre incapacité à conférer une dignité, dans la pleine dimension de leur altérité, à tous ces « non- » avec qui pourtant nous habitons ; à leur reconnaître et leur permettre la jouissance d'une vie bonne ${ }^{26}$. Elle me renvoie à Jacques Derrida qui pointe à la fin des années 1990 que nous vivons dans une société des " sans- »- les " sans-papiers » aux côtés desquels il se met en lutte, les "sans-abris", les "sans-domicile fixe ». Ce suffixe témoigne à ses yeux de l'insistance sociale à ce que tous et toutes se conforment, soient en conformité avec les attendus de la citoyenneté, avec les attendus de l'appartenance à la communauté (celui ou celle qui 
n'a pas de papiers s'en montrerait ainsi indigne). Faire pleinement communauté avec d'autres humains, avec des animaux, des molécules, des objets techniques, des divinités, des arbres, des bâtiments imposera donc que nous décentrions les perspectives afin de cesser de considérer l'autre comme celui ou celle qui devrait se conformer. Cesser également de considérer la nature comme ce «non-nous » qui nous sert d'arrière-plan. Alors peut-être, pourrons-nous commencer à transformer nos manières de faire relation et d'habiter ensemble, à envisager le choc que constitue la rencontre. Car il n'est pas sans difficulté d'être hôte, de recevoir autant que d'être reçu; cet "encaissement » dans tous les sens du terme que demande le vivre avec quand il se pense dans la durée ${ }^{27}$.

\section{Habiter la transformation}

- Tiphaine Abenia et Daniel Estevez. Les études de cas présentées dans le Pavillon français pour la Biennale posent la réutilisation, la transformation, la réparation et l'augmentation des existants comme des actes fondamentaux de la conception. Elles décrivent en cela les bases sociales et populaires d'une architecture non extractiviste (une architecture ne reposant plus sur l'extraction et l'exploitation de matières premières issues de régions conditionnées à leur production). L'extractivisme s'immisce dans les expulsions locatives, dans la privatisation de l'espace public, dans la raréfaction des matériaux de construction, dans la dégradation des territoires (pollution, surexploitation), dans la division du travail comme dans le rejet des communautés les plus vulnérables hors de la ville. Face à l'extraction, la pensée de la transformation pourrait-elle constituer un nouveau paradigme de la conception spatiale occidentale intégrant une prise en compte d'un temps long dans l'architecture?

Le sinologue François Jullien parle à ce sujet de la «modification-continuation » (biantong) par laquelle la pensée chinoise désigne les processus de transformation du cadre de vie : « La modification bifurque et la continuation poursuit, l'une innove, l'autre hérite. [...] c'est seulement à travers la modification que la continuation demeure active et qu'elle perdure $^{28}$. » Les architectures transformées, c'est-à-dire augmentées par l'usage, par I'habiter et par la construction matérielle positive, tracent-elles un avenir possible à une pensée durable et non extractiviste de l'urbain?

- Marion Howa. Sur ce sujet des transformations lentes, je voudrais à nouveau prendre l'exemple concret de la réhabilitation de la cité de Beutre que j'ai évoquée plus haut. En arrivant sur le site avec Christophe Hutin en 2019, nous avons constaté que la transformation des maisons avait commencé bien avant nous ; elle continue parallèlement au chantier que nous coordonnons, et elle se poursuivra une fois nos missions terminées. Par exemple, en deux mois, l'un des habitants a construit un très grand séjour qui s'étend vers le jardin et a doublé la surface de sa maison. Il aura poussé aussi loin que possible cet agrandissement. Si les panneaux de mur préfabriqués disponibles chez le fabricant avaient mesuré plus de six mètres, les dimensions de l'extension auraient certainement été encore plus grandes. Pour la lumière, il a prévu une baie vitrée sur le jardin, des puits de jour, une fenêtre de toit, des couleurs claires aux mur, plafond et sol. Ce séjour, qui évoque un open space, lui apporte plus d'avantages que l'usage d'un salon. Il y a suffisamment d'espace pour installer aussi l'activité professionnelle de sa compagne. En prévoyant une porte en attente sur la nouvelle façade qui donne vers le jardin, cet habitant a anticipé la construction future d'une chambre pour leur fille, qu'il réalisera plus tard. À l'heure où j'écris ces lignes, il construit une terrasse et envisage aussi de creuser une piscine dans le jardin. De la rénovation à l'agrandissement de sa maison, il aura tout fait de ses mains : gros-œuvre, démolition, charpente, isolation, 


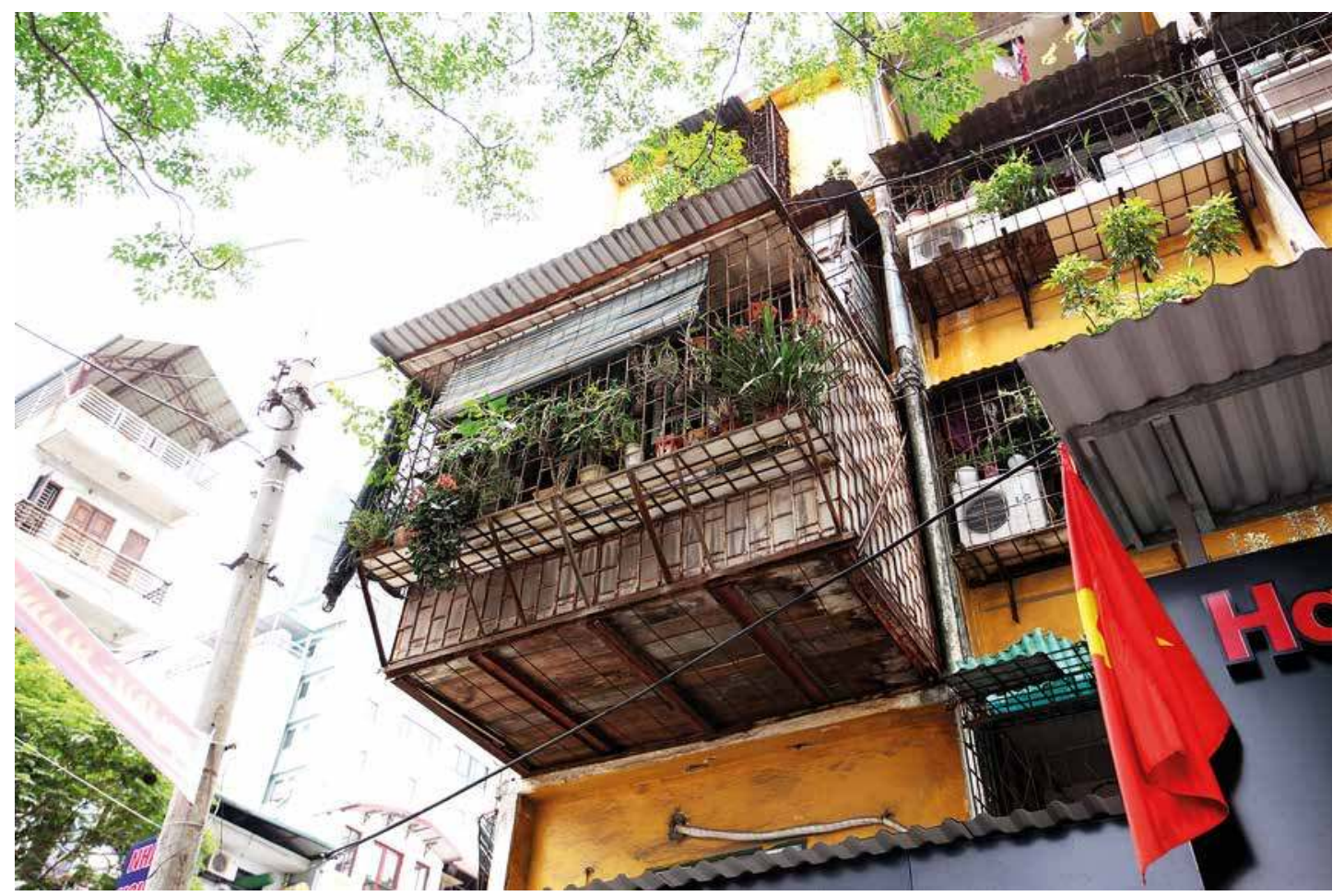

3a-b. Des modifications qui transfigurent les situations.

[a] Extensions dans un immeuble KTT (du vietnamien khu tập thể, " unités d'habitations collectives "), 2019, Hanoï. menuiseries, électricité, plomberie, sols, plâtreries, peinture. Même s'il boite depuis qu'il s'est fait opérer de la jambe, il a profité du temps de son arrêt maladie pour réaliser les travaux. II a reçu l'aide de son père, menuisier à la retraite, et de ses voisins de la cité, un groupe d'amis unis depuis l'enfance. En retour, il a rénové la salle de bains de l'un d'entre eux et posé la plâtrerie chez un autre.

Alors, de notre côté, nous nous sommes construit les outils sui generis pour comprendre et agir avec un tel processus d'évolution. Par « augmenter l'architecture », je désigne tout chantier qui accroît l'espace, le plaisir, l'usage et le pouvoir de l'habitant qui y vit ${ }^{29}$. À Beutre, ce processus architectural est manifeste : les faits sont observables quantitativement et qualitativement. Le diagnostic sociotechnique, I'approche diagrammatique du projet, l'écoute des quatre-vingt-treize familles au fil des journées passées avec les habitants sont autant de dispositifs qui documentent des actes, des structures, des relations, des besoins. L'hypothèse est que nous pouvons participer à la continuité de cette dynamique en prenant en compte la spécificité de chaque maison. À nous de savoir composer avec le mouvement, de répondre à des besoins bien réels et hétérogènes, de comprendre que formaliser un acte de construction d'un habitant peut protéger ou bien détruire la spontanéité, de repérer à quel endroit il est indispensable d'intervenir ou bien de laisser les choses se faire d'elles-mêmes.

- Tiphaine Abenia et Daniel Estevez. Cette situation rappelle celle de la ville informelle. Dans ce type de zone, le principe d'augmentation est très actif et favorise également les démarches de réutilisation des édifices. Cette pensée de la transformation perdure, et I'architecture incrémentale a été défendue, on s'en souvient, par Lucien et Simone Kroll. 
Or, c'est dans la ville informelle que les deux auteurs ont trouvé très tôt la figure intemporelle d'un urbanisme participatif populaire et vivant. Peut-on aller jusqu'à envisager cette dynamique des actions ponctuelles de la ville informelle comme une stratégie urbaine qui pourrait être implémentée à grande échelle?

- Fanny Léglise. II me semble que le plus important est la perspective selon laquelle nous regardons la ville informelle. Celle-ci peut être considérée comme une puissance urbaine pour peu que l'on adapte notre manière de l'observer, comme nous y invite David Edgerton. L'historien relève dans les bidonvilles des systèmes spécifiques et souvent novateurs de collecte des ordures, de fourniture d'eau, de nourriture, et répondant aux autres nécessités de la vie. En remettant en cause la définition traditionnelle du bidonville qui part d'un manque des équipements caractéristiques des villes riches, il développe une nouvelle compréhension de la "ville pauvre ", où il ne s'agit pas tant de se demander quelles techniques lui font défaut que de recenser celles dont elle dispose ${ }^{30}$. Les créations issues de la ville informelle ne relèveraient alors pas de "sous techniques ", mais d'une hybridation de mondes, puissamment féconde. Faut-il cependant envisager d'implémenter ces stratégies urbaines à grande échelle? Importer ou exporter des inventions ou techniques hybrides observées dans des pratiques informelles consisterait à nier leur puissante accroche à un contexte parce qu'elles se nourrissent du déjà-là et des habiletés et qualités de ses habitants. Et l'on risquerait peut-être de les employer comme une énième « formule d'aménagement » plaquée sur un ailleurs qui réfuterait la notion d'architecture, d'urbanisme, de design de la situation.

Ces stratégies semblent plutôt aptes à nourrir de nouveaux imaginaires lorsqu'elles sont diffusées ailleurs, probablement porteuses d'un fort pouvoir de déplacement par une nouvelle opération de transformation. Par la transmission de leur récit, elles peuvent faire naître de nouvelles façons de procéder. Le sociologue du design Ezio Manzini relève dans la cohabitation de pratiques autonomes et hétéronomes qu'un " nombre croissant de personnes est appelé à utiliser sa capacité "naturelle" de conception de façon exponentielle, parce que la phrase "nous sommes tous designers" ne se réfère plus à une potentialité mais à une réalité à laquelle nous devons faire face ${ }^{31}$ ». Dans ce cadre, plusieurs types de savoirs, notamment experts et non experts, se rencontrent. Pour que tout le monde puisse travailler ensemble, le chercheur estime qu'il faut des plateformes digitales, des espaces physiques de rencontres, des services logistiques, d'information, de communication, une infrastructure. Nous pourrions y ajouter le recours à des médiateurs ou des facilitateurs qui traduiraient et transmettraient ces récits d'une situation à l'autre. L'exposition au Pavillon français que vous avez organisée avec Christophe Hutin se situe dans la lignée de cette démarche.

Ce qui nous intéresse finalement ne seraitil pas plutôt cette recherche d'une forme de cohabitation entre plusieurs espèces d'architectures ou de systèmes urbains? Je pense ici au travail du philosophe Baptiste Morizot ${ }^{32}$ autour des notions de perspectivisme ${ }^{33}$ et de diplomatie. Ne devrions-nous pas nous attacher à " voir comme un habitant », à " penser comme un bricoleur ", dans une attitude ouverte qui

[b] Transformation d'une flaque d'eau en bassin à Détroit (quartier du South West), 2013.

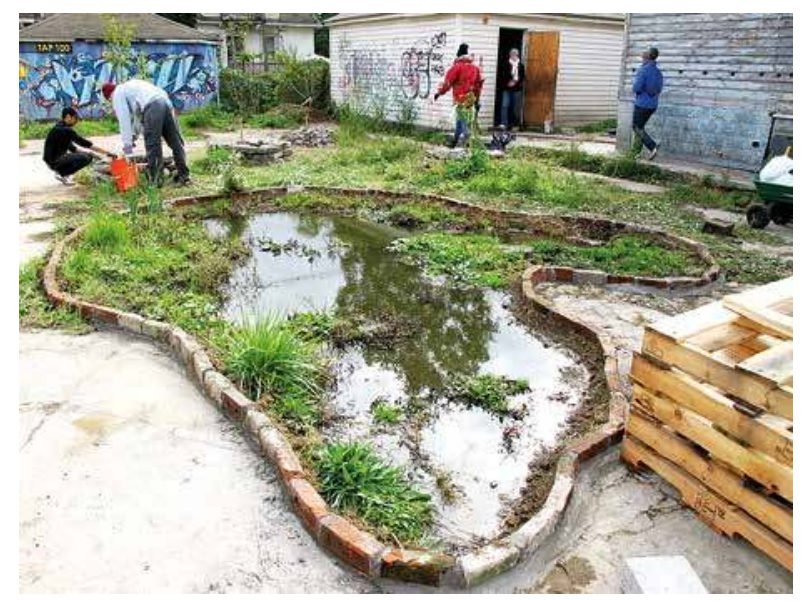


laisserait toutes les tactiques possibles coexister? Ne serions-nous pas plus riches de possibilités si nous profitions de la médiation de diplomates-architectes-habitants capables de traduire des façons d'agir a priori très éloignés ? Ces derniers permettraient certainement de garantir que les rencontres entre « experts » et « non-experts » soient fructueuses. N'aurions-nous pas tout intérêt à ajuster normes et contraintes aux situations, de façon à nous libérer du rôle de prescripteur (de matériaux neufs, intimement liés à l'extraction) pour rejoindre celui d'interprète (de matériaux déjà œuvrés, aptes à la transformation) ? L'architecte ne pourra jamais concevoir des habitats informels. Leurs habitants ne demanderont jamais à ce dernier de dessiner leur logement ; cependant ces deux mondes peuvent se rencontrer et s'enrichir, mus par la curiosité.

\section{Habiter / bricoler}

- Tiphaine Abenia et Daniel Estevez. Vous évoquez ici le bricoleur lévi-straussien qui "fabrique des mondes " à travers son action concrète située et à partir d'un ensemble "à tout instant fini » de moyens disponibles ${ }^{34}$. Cette figure semble comporter en effet de nombreuses analogies avec celle de I'habitant agissant. L'une des hypothèses des travaux présentés dans le Pavillon français

4a-b. Agir avec les moyens disponibles.

[a] Salle de répétition dans la maternité désaffectée Florence House, Johannesburg, atelier « Learning from » (ENSA Toulouse), 2011-2010.

[b] Cantine communautaire dans l'hôpital désaffecté Elefante Blanco, à Buenos Aires, 2013.
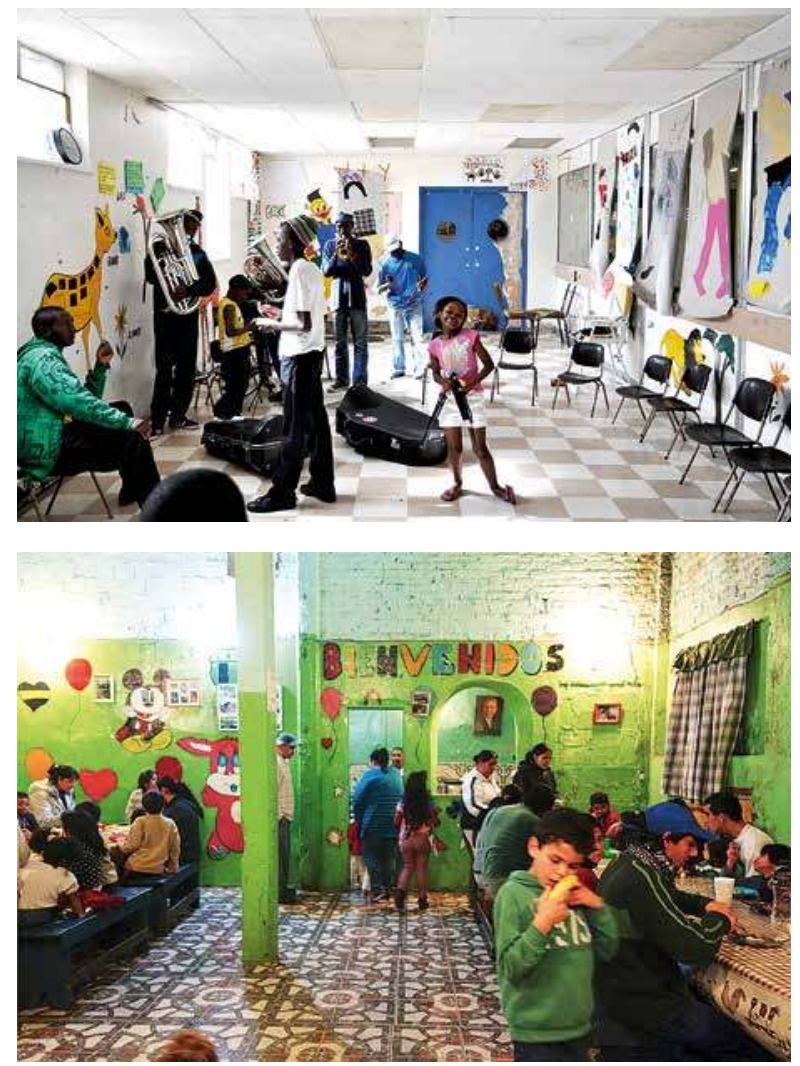
de la XVII Biennale d'architecture de Venise postule que l'étude des productions bricolées des habitants peut permettre d'accéder à une connaissance théorique valide de la conception spatiale en général. On envisage alors la conception architecturale et urbaine dans sa réalité humaine et sociale, mais aussi intersubjective et technique en considérant avec Bernard Rudofsky que l'architecture est un phénomène anthropologique puissant qui déborde toujours les savoirs formalisés a priori par les architectes. L'efficacité du bricolage repose sur une attention active portée par chaque sujet aux moyens existants, aux ressources dont il dispose pour agir sans en exiger de nouvelles. La première leçon que nous pouvons certainement tirer des situations vernaculaires contemporaines observées est ainsi celle de l'efficience des moyens dans l'action : "C'est un principe d'économie : avec le minimum de forces, obtenir le maximum d'effets. Il définit une esthétique, on le sait ${ }^{35}$. » Pourtant, l'univers d'invention du bricoleur n'est-il pas saturé de précédents, de cas, de choses déjà vues ?

- Fanny Léglise. La ville comme bricolage est une image récurrente que l'on trouve dans des écrits qui la définissent comme un palimpseste, une rencontre impromptue entre des mondes, des modes, des époques ou un 
tissu organique en perpétuel mouvement ${ }^{36}$. Quant au concepteur-bricoleur ${ }^{37}$, il correspond à une figure qui interroge, depuis l'anthropologie jusqu'à l'architecture et bien d'autres disciplines. L'encyclopédisation ${ }^{38}$ des pratiques de conception alternatives est à l'œuvre depuis les années 1960 sous toutes les formes : inventaires, catalogues, manuels, bases de données numériques, tentatives de théorisation. Ces autres manières de faire montrent comment les négociations, la ténacité, l'imagination, les rencontres participatives et la vision de chacun en tant qu'acteur peuvent porter une compréhension différente des pratiques architecturales et urbaines en tirant des leçons critiques, aussi bien vis-à-vis des politiques, de la profession, de la pédagogie que des crises. Le bricolage est issu d'une philosophie du détournement qui s'oppose à la planification : il n'a ni théorie, ni agenda, ni échelle de prédilection ou tradition formelle, intellectuelle. Il s'applique à des réalités mouvantes, déconcertantes et ambiguës qui font probablement sa force dans un monde incertain.

L'inséparabilité et l'alliance entre concepteur et situation dans le cadre du bricolage interrogent sur la finitude des ressources et la façon dont nous considérons les employer, s'éloignant de l'extractivisme issu des sociétés industrielles. Le stock lévi-straussien, «à tout instant fini », composé à partir des moyens du bord, engage à repenser les transferts entre objet, déchet et nouvel objet. La « rematérialisation » caractéristique du bricolage véhicule une façon de changer la société et les rapports humains, et non uniquement le rapport à la construction ou à l'objet. Cette rematérialisation engage une forme de "réoutillage », au sens que lui donne le philosophe Ivan Illich, à comprendre tant au sens physique qu'intellectuel. Elle incarne très probablement une note optimiste et facétieuse dans un monde en crise. Malgré la multitude d'initiatives qui vont dans le sens du bricolage, elles ne semblent jamais épuisées : peut-être celui-ci se nourrit-il justement de la saturation?

\section{Habiter des structures communes}

- Tiphaine Abenia et Daniel Estevez. ...Et du désordre ? Dans son texte « La tyrannie de l'absence de structure ", diffusé à la fin des années 1970, l'activiste féministe américaine Jo Freeman se demandait comment agir sans s'entraver dans des structures aliénantes, et s'il est possible de se passer de structure ${ }^{39}$. Sa réflexion concernait les possibilités d'une organisation radicalement démocratique des actions collectives et s'appuyait sur ses expériences menées dans le domaine de la lutte des femmes pour la reconnaissance de leurs droits. Jo Freeman dresse un bilan négatif des possibilités d'action collective portée par le refus de tout principe organisateur. Cette observation trouve un puissant écho dans la pensée structuraliste en architecture, laquelle soutient le développement de structures collectives aux principes organisationnels forts, stables et intelligibles. Résistantes car sans cesse changeantes, de telles structures tirent leur pertinence de leur capacité à répondre à l'incertitude de notre monde en considérant l'imprédictibilité des besoins et la raréfaction des ressources.

Dans un autre domaine, celui de l'improvisation musicale contemporaine, vous dressez, Christopher Dell, le même type de constat. Vous affirmez notamment que le désordre et l'absence de structure ne sont pas la garantie d'une improvisation créative et pertinente. Faisant le lien avec la création architecturale, vos propos entrent étonnamment en écho avec ceux de Jo Freeman quand vous dites : "On ne doit pas être victime de l'improvisation. L'improvisation suppose l'existence d'un plan mais cherche à le transgresser par la performance $^{40}$. » En quoi peut-on considérer l'habiter comme une performance sociale analogue à celle de l'improvisation musicale que vous décrivez?

- Christopher Dell. Une ville est un relais de structures bâties, de technologies de régulation, d'entités légales, et elle est également composée d'acteurs, d'actions, d'imaginaires et de 


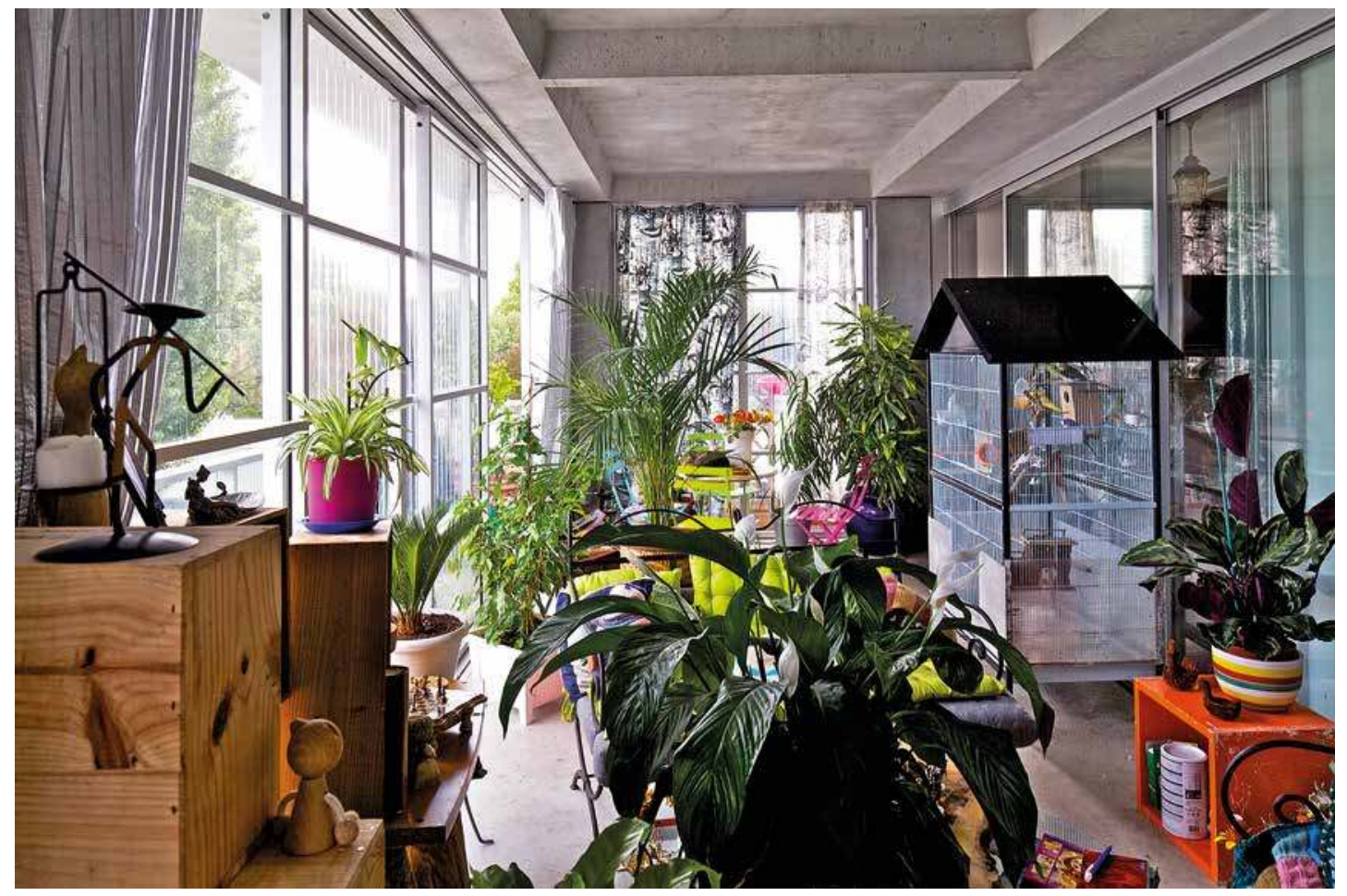

5a-b. Appropriations en situation formelle ou informelle.

[a] Un jardin d'hiver, projet GHI (Anne Lacaton et Jean-Philippe Vassal, Frédéric Druot et Christophe Hutin), réhabilitation de la cité du Grand Parc, à Bordeaux, 2016. mémoires. C'est l'interaction contingente de tous ces éléments avec les choses qui produit l'espace urbain. Plutôt que de réduire la ville à des dichotomies - formel versus informel, visible versus invisible, objectif versus subjectif, agencement versus structure - s'impose la nécessité pour l'architecture et le design urbain d'envisager une perspective plus complexe sur la

ville et son habitat. II nous faut abandonner ici l'alternative entre acte libre et déterminisme. La capacité d'agir est toujours située ; elle n'est jamais définitivement constituée. C'est la qualité performative des acteurs et des objets de la ville, leur fabrication et refabrication, le raccordement et le réassemblage de ses parties qui constitue la qualité d'improvisation de la ville.

Si l'on pense à un coin de rue avec un kiosque, un arrêt d'autobus et un banc, avec des usagers qui activent cette situation urbaine de différentes manières, il devient évident que les options structurelles offertes par la matérialité de la situation affectent ce qui se passe. Le kiosque va prendre du sens en fonction de ce que vous en faites : c'est-à-dire que vous pouvez y acheter un journal (usage consumériste), discuter avec le vendeur (usage social), ou bien utiliser ses services internet (usage de communication), ou encore y récupérer un colis parce que vous n'étiez pas chez vous lors de la distribution (usage de stockage). Cet exemple montre à l'évidence que les relata (les humains et les choses qui constituent la situation urbaine) n'existent pas avant les relations desquelles ils participent. II n'existe pas d'extérieur à la situation urbaine.

Dans ce sens, le matériel agit aussi. La matérialité des choses, des corps, des actions et des discours est constituée de la reconfiguration constante et permanente des structures de la ville, qu'elle affecte. Nous interprétons nos actions rétroactivement. La discursivité 
et la matérialité de l'habitation quotidienne sont coextensives; elles constituent un diagramme qui est improvisé par les acteurs et les choses de la ville. Tous les corps - ce qui n'implique pas seulement les êtres humains, mais aussi les corps non humains, tels que les immeubles, les rues, les îlots d'habitation, les murs, les massifs, etc. - acquièrent leur poids dans le monde à travers une telle improvisation. La matérialité de l'inscription spatiale n'est pas une assignation mais une improvisation au sens de technologie. J'ai défini ainsi cette improvisation : «travailler de manière constructive avec la contingence au sein d'un assemblage d'acteurs, de choses,

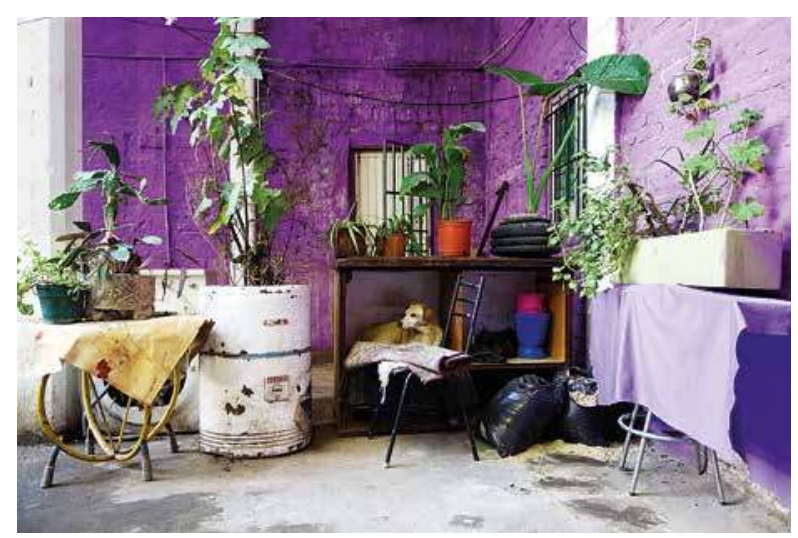

[b] Entrée d'un appartement dans l'hôpital squatté Elefante Blanco, à Buenos Aires, 2013. d'actions et de discours sur un site ${ }^{41} »$. Adopter cette approche pour comprendre la matérialité comme un ensemble d'aménagements, ou un assemblage de choses et d'êtres humains, permet de passer du design architectural comme tabula rasa à une lecture des situations sous l'angle de structures qui peuvent être réassemblées d'une nouvelle manière.

Cette perspective inclut également l'investigation de l'architecture et du design urbain dans leur propre matérialité conceptuelle, c'est-à-dire leurs techniques et leurs stratégies de visualisation ${ }^{42}$. C'est la raison pour laquelle je tiens à faire la différence entre improvisation réflexive et non réflexive, c'est-à-dire entre l'improvisation comme mode de réparation et l'improvisation comme technologie ${ }^{43}$. Cette dernière ne se fait pas sans plans, ni à leur encontre, mais elle permet de les faire évoluer vers une pratique conceptuelle située. C'est seulement à partir de là que l'architecture et le design urbain, en tant que pratiques de lecture, d'exposition, de négociation et de construction d'identités matérielles de la ville, peuvent devenir à la fois une improvisation technologique et les conditions d'existence de celle-ci.

\section{La technicité des milieux}

- Tiphaine Abenia et Daniel Estevez. Le fait d'envisager l'improvisation sociale comme une technologie d'agencement qui n'est pas indépendante de la constitution matérielle des milieux de vie nous amène à la question technique. L'habiter a parfois été décrit, notamment par des auteurs comme Madeleine Akrich ou Philippe Descola, comme un processus sociotechnique d'aménagement matériel par les groupements humains de leur environnement familier. Or, ce processus se voit aujourd'hui contrarié par certains modes d'équipement technologique de notre cadre de vie, marqué par une automatisation croissante. Les technologies de l'automatisme tendraient en effet à diminuer l'action directe de l'habitant sur son milieu entraînant des effets de désimplication sociale ; l'improvisation s'éloigne. Gilbert Simondon lui-même avait insisté sur les faiblesses techniques que recèle l'automatisme dans son principe, celui-ci relevant en fait d'un degré assez bas de perfection technique. Le philosophe Bernard Stiegler proposait quant à lui le concept de " prolétarisation » pour décrire cette dépossession de nos compétences à travers l'équipement technologique automatisé de notre environnement. Dans une société-machine ${ }^{44}$, I'automatisation gagne du terrain sur l'action directe humaine et, par là même, la déqualifie. En tant que phénomène d'activité sociale, l'habiter reposerait donc en partie sur l'existence d'un environnement technique 

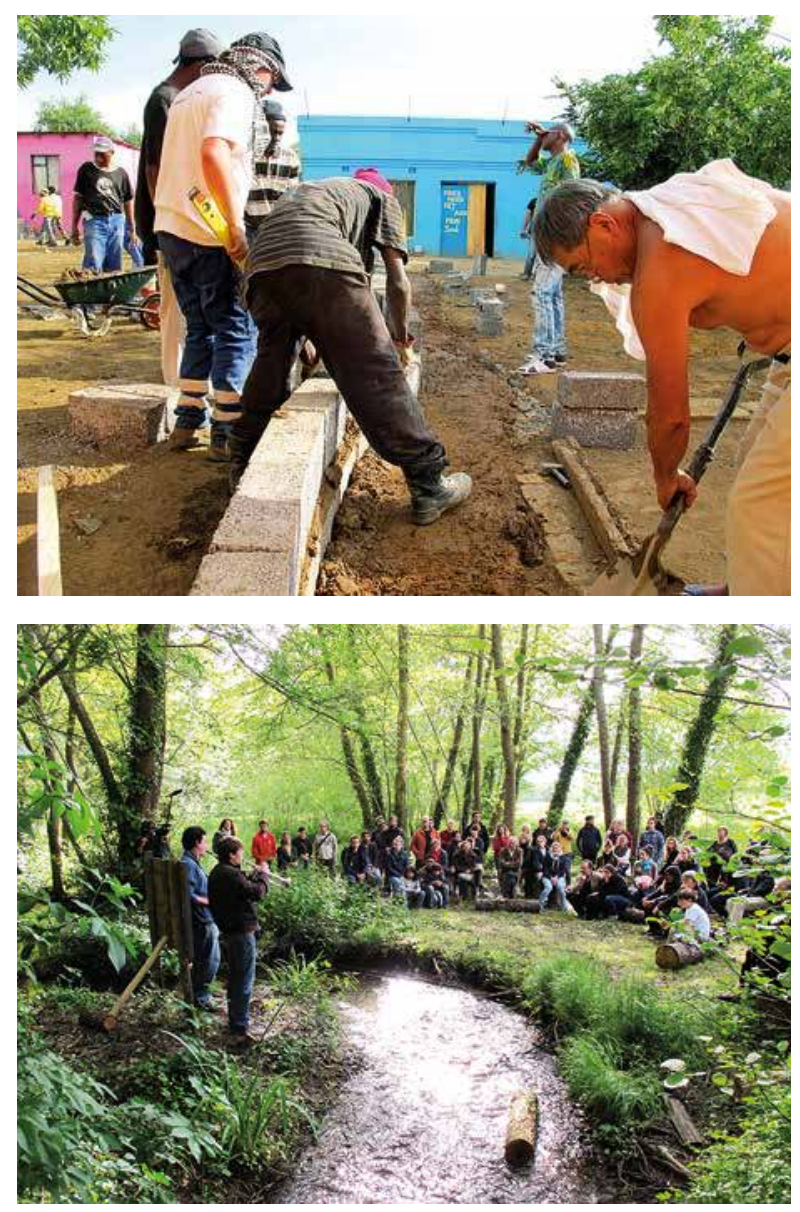

6a-b. L'improvisation comme technique d'agencement. [a] Construction du mur serpent à l'orphelinat Soweto Kliptown Youth à Soweto, atelier "Learning from » (ENSA Toulouse), 2012.

[b] Improvisation aux abords du ruisseau d'Uzeste (Gironde), atelier « Learning from » (ENSA Toulouse), 2013.

stimulant parce qu'indéterminé dans son fonctionnement. Dans la dépossession et la désimplication aujourd'hui observées, nous retrouvons certains rouages des démarches de conception extractiviste qui ont pour conséquence de déposséder les sujets (concepteurs, habitants) de leur capacité d'action (autonomie, socialisation, compétences). Pouvons-nous dès lors envisager un milieu technique qui soit compatible avec la vie sociale?

- Matthieu Duperrex. Je pense que, depuis toujours, on habite par les milieux. Mais votre question pourrait se formuler autrement : "Comment pouvons-nous aimer nos techniques, réellement les aimer?", demandait Bruno Latour dans son exposition "Reset Modernity! ${ }^{45}$ ». En déployant de nouvelles alliances, en habitant de nouvelles pellicules protectrices (Umwelten), desquelles prendre soin. Le front de modernisation du capitalisme s'est développé au moyen de stratégies d'invisibilisation des infrastructures et des réseaux de pouvoir, c'est particulièrement vrai pour les industries carbonées ${ }^{46}$. Or, ainsi que le montrent les études que les sciences sociales consacrent aux thèmes de la maintenance et du soin, la préservation de notre technosphère ${ }^{47}$ passe par la mise en évidence de ses fragilités et systèmes de dépendance ainsi que par le réinvestissement de la durée du « travail vivant » au cœur du système productif.

Je soutiens que cette durée du travail vivant, que Marx avait thématisée dans ses manuscrits (Grundrisse) de 1857-1858, doit être évaluée en y intégrant la part des nonhumains et des milieux, bien qu'à rebours d'une économisation de la nature qui n'identifie que des «services écosystémiques" pour des usages exclusivement anthropocentriques ${ }^{48}$. Lorsqu'en 1958 le philosophe Gilbert Simondon, que vous citez, subordonnait l'innovation ou la « concrétisation » de l'objet technique à la naissance d'un « milieu associé », hybride entre milieu " géographique » et milieu " technique ", il plaidait en définitive pour une autonomisation et une individualisation de la techno-sphère au voisinage d'une conception beaucoup trop homogène et indifférenciée de la « nature ${ }^{49}$ ». Habiter " par les milieux », c'est reconnaître au contraire le pluralisme ontologique qui est irréductible à cette homogénéisation de la nature et saisir en quoi, et par quels véhicules, les réseaux sociotechniques enrôlent humains et non-humains dans la confection de nos enveloppes habitées. Un chantier prospectif s'ouvre alors pour définir son dû au travail du vivant, des vivants, dans leurs liens constants avec les formes du monde, avec les sols et l'air, et les cycles de l'eau. 


\section{Habiter dans l'hétérogénéité}

- Tiphaine Abenia et Daniel Estevez. Le Pavillon français de la Biennale de Venise s'inscrit dans la filiation intellectuelle de la pensée décoloniale du poète Édouard Glissant. Sa méditation sur la plantation nous informe encore. Lieu clos et isolé, la plantation chez Glissant rejoint la formule sociospatiale contemporaine du camp (de rétention, de concentration, d'internement, de transit, de réfugiés...). Historiquement, Glissant analyse la plantation comme un système d'oppression et de fixité qui contraint les dominés à la bifurcation, au subreptice, au fragment, à la forme brève, à l'esquive. Pour lui, les conditions de résistance d'une vie d'oppression ont toujours à voir avec le discontinu et l'hétérogène. Il s'agissait aussi de reconnaître une pensée de la multiplicité qui permettrait de construire des outils, des actions $d^{\prime}$ '" empuissantement ». À l'isolement du camp Glissant va ainsi notamment répondre par la figure ouverte de l'archipel, une pensée rhizomatique permettant à des identités singulières de se rencontrer, de s'entrechoquer et de produire du nouveau.

Les réflexions de Glissant nous enjoignent de chercher ces nouveaux points d'appui, ces poches localisées et plurielles faisant archipel. Peut-on subvertir aujourd'hui la formule sociospatiale du camp (unicité, uniformité, coercition) ? Comment imaginer un contre-modèle?

- Matthieu Duperrex. Zads, squats, mouvement néo-paysan, insurrection indigène, etc., mais aussi écologie critique, bio-régionalisme, animisme juridique... - nombre de luttes contemporaines ${ }^{50}$ témoignent de l'idée qu'il ne nous reste plus qu'à investir et habiter des " archipels » où résister à l'accaparement des lieux de vie par cette simplification et exploitation de l'espace dont la plantation coloniale est l'archétype et dont le capitalisme poursuit l'entreprise de mise à l'échelle ${ }^{51}$. Dès le fameux Et in Arcadia ego

7a-b. Multiplicités dans les milieux vivants. [a] Voisinages des bacs potagers naturels, réhabilitation à Beutre, 2019.

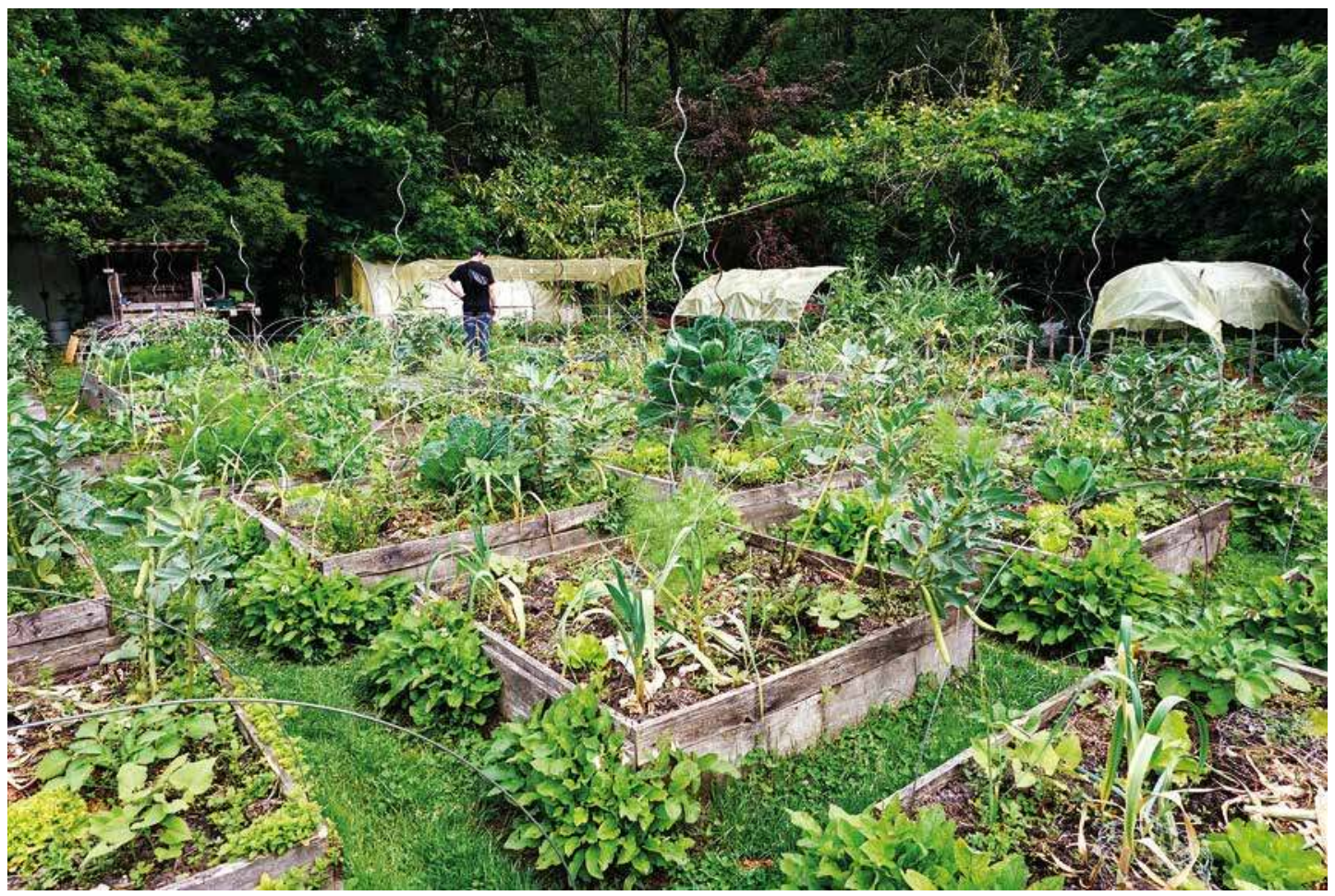




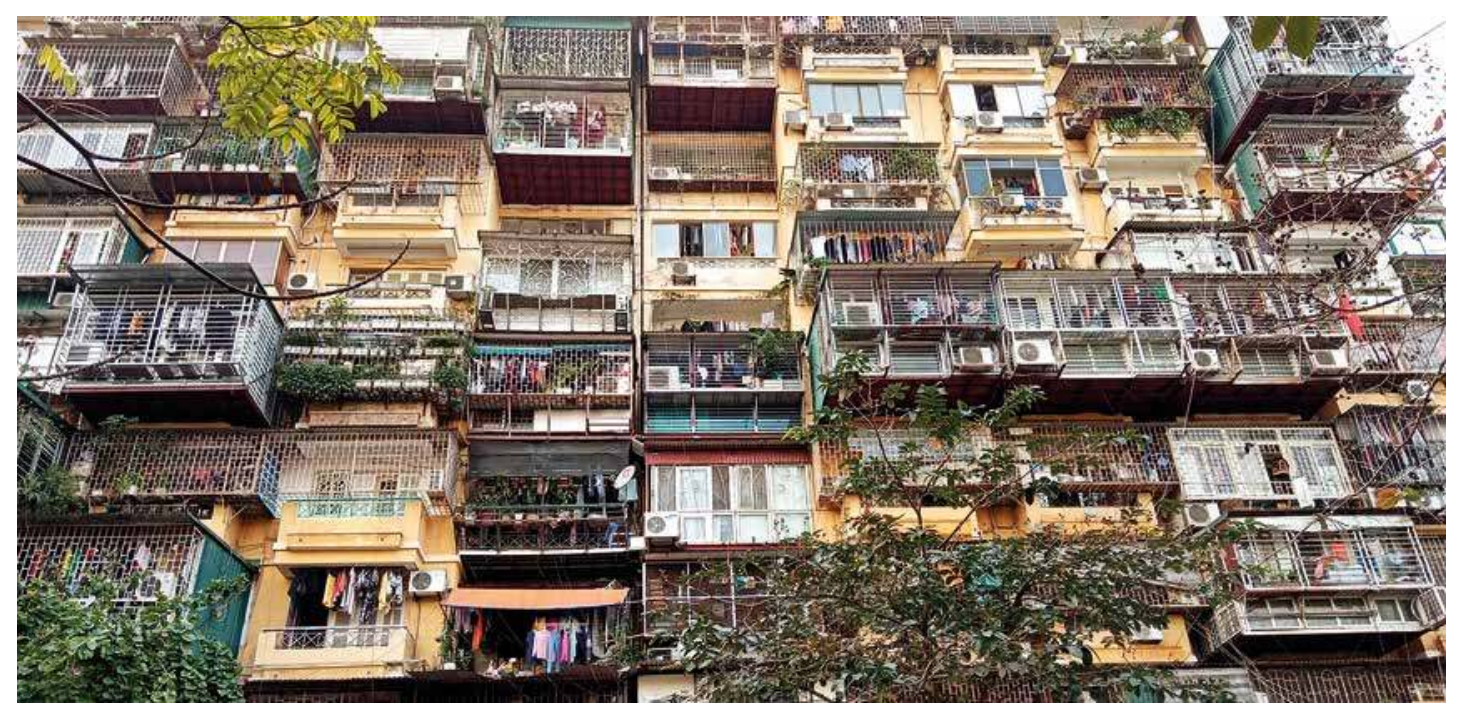

[b] Extensions dans un immeuble KTT, que déchiffrent les bergers d'Arcadie sur le tombeau, dans 2019, Hanoï.

le tableau de Nicolas Poussin (1640), I'Arcadie est une pastorale affectée par une menace sourde et incorporelle que nous tentons d'élucider. En effet, ce sont depuis des patchs de souveraineté qu'un art démocratique de l'enquête sur nos attachements, dépendances et modes de subsistance peut s'opposer aux processus de colonisation, de mise à l'échelle et de captation.

Dans le contexte qui est celui de l'anthropocène, j'appelle « Arcadie altérée » ce lieu du déchiffrement, des sédimentations et recouvrements de référents - techniques, géologiques, végétaux, animaux, humains - à l'intérieur de la pellicule éco-techno-symbolique de l'écoumène (soit de l'ensemble des milieux habitables sur la surface terrestre) en déliquescence. L'Arcadie est un refuge, c'est un lieu par définition liminaire, qui se révèle d'autant plus plein et habité que l'enquête progresse. Mais l'Arcadie surnage dans l'océan entropique de I'anthropocène, œuvre de déliaison et de destruction. Aussi, pour les lieux habités comme pour les entités qui y vivent, l'altération est plurimodale : comme précarité de notre condition et de nos ajustements à l'environnement, comme incertitude de la relation à une "nature » contaminée par les hybrides, comme distorsion d'un schème de représentation / perception dans lequel on avait placé sa confiance pour évaluer la situation, comme faillite des temporalités instituantes du pacte social et de leurs prises sur le monde réel, avec ce doute quant à l'allure de ce qui est en fait une conduite « réaliste »... II nous incombe aujourd'hui d'habiter une Arcadie altérée.

- Tiphaine Abenia et Daniel Estevez. Mais ces altérations des milieux de vie ne définissentelles pas aussi des lieux où atterrir? L'économiste Saskia Sassen écrit :

Quels sont les espaces des expulsés ? Ces espaces sont invisibles d'après les mesures courantes. Économie réduite en Grèce, élites prédatrices en Angola, augmentation des chômeurs et des prisonniers aux États-Unis, l'espace des expulsés grandit. De même, la prolifération des terres mortes liées à la déforestation, à l'industrie des biocarburants, etc. Ce sont des situations souterraines, d'un point de vue conceptuel, qui ont besoin d'être ramenées à la surface. De façon générale, les espaces des expulsés réclament une reconnaissance. Ils sont potentiellement les nouveaux espaces où il est possible de créer - créer des économies locales, créer des histoires nouvelles, créer de nouvelles modalités d'adhésion ${ }^{52}$. 
À chaque fois qu'une situation critique existe, ne peut-on y voir avec Sassen un point d'appui réel pour une adhésion nouvelle, pour une résistance nouvelle?

- Christopher Dell. Envisageons votre question sous l'angle de l'improvisation. Spécifiquement - plus qu'une simple théorisation de la ville comme processus - je soutiens qu'une réévaluation épistémologique de l'expérience urbaine à travers une réorientation de la compréhension de l'action elle-même est nécessaire. Cette position privilégie une nouvelle orientation dans laquelle l'action n'est plus l'aspect rétrospectif d'un plan ou une opposition à la structure, mais plutôt - interprétée comme une improvisation - un processus technologique opérationnel décisif, structurel en soi, dans la fabrication de la ville. Dans un sens politique, cela revient à élargir l'horizon vers un universalisme ouvert, où les effets de la domination - pas formellement mais structurellement - contiendront les moyens de leur résister.

C'est pourquoi le concept d'improvisation est pertinent pour comprendre les « nouvelles adhésions » que vous évoquez. Lire l'espace en tant qu'espace improvisé éclaire sa qualité essentiellement démocratique. Pour le dire simplement, on ne peut pas ne pas improviser l'espace. Tout le monde le fait. L'improvisation de l'espace est un déterminant basique de l'existence urbaine. Cependant, à l'encontre d'une idéologie néolibérale de l'individu flexible, j'avance la thèse que les acteurs urbains n'agissent pas sans les conditions de l'agir ; et, paradoxalement, c'est précisément la configuration de ces conditions elles-mêmes que I'on doit parfois installer ou préserver afin de pouvoir agir. Constituant un mode d'action contextuel, l'improvisation est ancrée dans un ethos, ce dernier étant compris ici dans le sens existentialiste d'un lieu d'habitation, d'une manière de s'orienter dans une situation urbaine et de l'habiter. À cet égard, l'improvisation est une manière active d'envisager la ville, de la comprendre et de la regarder. C'est une manière de se déterritorialiser et de se reterritorialiser dans la réalisation et la perception de la ville, et d'accepter le rôle fondamental que joue la contingence dans ce processus. Je soutiens donc que l'éthique est toujours impliquée, non seulement dans les questions économiques et sociales, mais aussi dans les questions spatiales. Aujourd'hui, alors que les formes d'habiter, de vivre et de travailler semblent se confondre socialement et spatialement, la vie que l'on mène est toujours une vie urbaine. Concevoir l'action comme improvisation indique - considérant la manière d'agir dans la vie urbaine - que l'on fait implicitement référence aux conditions de la ville comme permettant d'agir dans des conditions d'indétermination.

Ce que j'appelle improvisational lens (que l'on pourrait traduire par « perspective improvisationnelle ») constitue un domaine traversé par diverses forces motrices qui tendent à redéfinir et à renégocier le sens de l'action par rapport à ses propres conditions spatiales. Ces forces soulignent à la fois l'aspect temporel et séquentiel de la formation de la ville, l'engagement politique et éthique qui y est impliqué, et sa spatialisation. Dans les recherches sur la question de la ville, et son traitement, il semble nécessaire de mettre de côté les solutions préfabriquées à des problèmes identifiés comme donnés ou acquis, pour se concentrer sur la conception d'agencements improvisés de l'habiter, qui permettraient aux questions d'émerger et dans lesquels la res (chose) redeviendrait publica (publique).

Les propos de Christopher Dell ont été traduits

de I'anglais par Michèle Veubret. 


\section{Tiphaine Abenia}

Tiphaine Abenia est ingénieure en génie civil, architecte et docteure en architecture. Ses recherches portent sur les phénomènes urbains liminaux, les structures ouvertes en architecture et la catégorisation comme outil de conception critique du projet. Elle enseigne, depuis 2019, le projet d'architecture et l'enquête par le dessin à l'École polytechnique fédérale de Lausanne (Suisse).

\section{Mathilde Chénin}

Mathilde Chénin est artiste. Elle est cofondatrice du projet " bermuda ", lieu mutualisé de vie et de travail artistique situé à la frontière francosuisse (bermuda.pm). Doctorante en arts visuels et sociologie urbaine, elle termine sa thèse de doctorat, intitulée «Quand les artistes font forme en habitant ensemble » (HEAD - Genève, Lasur / EPFL, 2022).

\section{Christopher Dell}

Christopher Dell est musicien, docteur en philosophie et urbaniste. Il travaille et enseigne à Berlin en tant que théoricien de l'aménagement urbain et de l'architecture, mais aussi en tant qu'artiste et compositeur. Il a été professeur de théorie de l'aménagement urbain à l'université HafenCity de Hambourg, à I'université technique de Munich et à l'université des arts de Berlin. Il a publié de nombreux ouvrages notamment La Ville comme partition ouverte (Zürich, Lars Müller, 2016) et The Improvisation of Space (Berlin, Jovis, 2019).

\section{Matthieu Duperrex}

Matthieu Duperrex est philosophe, il enseigne les sciences humaines à l'École nationale supérieure d'architecture de Marseille. Artiste et théoricien, il est aussi directeur artistique du collectif « Urbain, trop urbain " (www.urbain-trop-urbain.fr). Ses travaux procèdent d'enquêtes de terrain sur des milieux anthropisés et croisent littérature, sciences humaines et arts visuels. Il est notamment l'auteur de Voyages en sol incertain (Marseille, Wildproject, 2019), qui prend la forme d'une enquête dans les deltas du Rhône et du Mississippi.

\section{Daniel Estevez}

Daniel Estevez est architecte, ingénieur en informatique fondamentale et professeur HDR à l'École nationale supérieure d'architecture de Toulouse. Il est également chercheur et coordinateur du groupe «Art, Architecture et Conception » au laboratoire de Recherche en architecture de I'ENSA Toulouse. Depuis 1995, son travail scientifique concerne l'étude des pratiques contemporaines de conception en architecture dans le contexte du numérique mais aussi en milieu critique.

\section{Marion Howa}

Marion Howa est architecte, diplômée en sciences politiques et enseignante-chercheuse vacataire à l'ENSA Toulouse. Doctorante en conventions industrielles de formation par la recherche (CIFRE), elle travaille depuis 2013 avec Christophe Hutin. Elle est coauteure du catalogue de l'exposition du Pavillon français. Sa recherche doctorale porte sur les possibilités d'action de conception des architectes à travers les outils issus des théories pragmatistes et de la transformation sociale.

\section{Fanny Léglise}

Fanny Léglise est architecte et docteure en architecture. De 2009 à 2015, elle assure la coordination éditoriale puis la rédaction en chef de L'Architecture d'aujourd'hui. Elle développe depuis 2016 une pratique indépendante au service des métiers de la ville en tant que chercheuse, auteure et commissaire d'expositions.

\section{NOTES}

1. Édouard Glissant, La Cohée du Lamentin, Paris, Gallimard, 2005, p. 139

2. Christophe Hutin (dir.), Les Communautés à l'œuvre / Communities at Work, cat. exp. (Venise, Pavillon français de la XVII exposition internationale d'architecture - La Biennale di Venezia, 2021), Paris, Carré, 2021.

3. Donald A. Schön, The Reflective Practionner. How Professionals Think in Action, New York, Basic Books, 1983, p. 76

4. Jacques Rancière, «Les mésaventures de la pensée critique ", Le Spectateur émancipé, Paris, La Fabrique, 2008, p. 85.

5. Ibid., p. 55.

6. Voir Chimères. Revue des schizoanalyses, fondée par Gilles Deleuze et Félix Guattari, «Politiques de la communauté », n 87, février 2016.

7. Des plumes dans le goudron, Résister aux grands projets inutiles et imposés. De Notre-Dame-des-Landes à Bure, Paris, Textuel, 2018, p. 122.

8. Laura Centemeri, "Entre expertises et émotions: les conflits relatifs à l'extension de l'aéroport de Milan Malpensa ", dans Christophe Traïni (dir.), Émotions et expertises : les modes de coordination des actions collectives, Rennes, Presses universitaires de Rennes, 2015, p. 127-146 [URL : https://hal.archives-ouvertes.fr/hal01077265v2/document].

9. Christian Norberg-Schulz, Genius Loci. Paysage, ambiance, architecture, Bruxelles, Margada, 1981.

10. Jacques Rancière, Le Maître ignorant, Paris, Fayard, 1987, p. 26.

11. Marie Hélène Bacqué, Carole Biewener, L'Empowerment, une pratique émancipatrice?, Paris, La Découverte, 2015. 
12. Judith Butler, Trouble dans le genre. Pour un féminisme de la subversion, Cynthia Kraus (trad. fr.), Paris, La Découverte, 2005 [éd. orig. : Gender Trouble, Feminism and the Politics of Subversion, New York, Routledge, 1990].

13. Voir Jacques Dars, Les Carnets secrets de Li You, Arles, Picquier, 2009.

14. Voir Henri Lefebvre, La Production de l'espace, Paris, Anthropos, 2000, p. 253.

15. John F. C. Turner, Housing by People: Towards Autonomy in Building Environments (1977), New York, Marion Boyars, 2000, p. 66.

16. Édouard Glissant, Poétique de la Relation, Paris, Gallimard, 1990, p. 109.

17. Olivier Thierry, Sophie Houdart, "Avant-Propos", dans Sophie Houdart, Olivier Thierry (dir.), Humains, non-humains. Comment repeupler les sciences sociales, Paris, La Découverte, 2011, p. 7-13, ici p. 7.

18. Nicolas Bourriaud, Esthétique relationnelle, Dijon, Les Presses du réel, 1998.

19. Voir Bruno Latour, Où atterrir ? Comment s'orienter en politique, Paris, La Découverte, 2017.

20. Voir Bourriaud, 1998, cité n. 18.

21. Voir François Bottelier Depois, "Fablabs, Makerspaces : entre nouvelles formes d'innovation et militantisme libertaire ", Observatoire du Management Alternatif, Cahier de recherche, HEC Paris, 2012.

22. Voir Claire Bishop, "Antagonism and Relational Aesthetics", October, vol. 110, 2004, p. 51-79.

23. Voir Chantal Mouffe, "Artistic Activism and Agonistic Spaces ", Art and Research, vol. 1, n 2, été 2007.

24. Livio Boni, Sophie Mendelsohn, La Vie psychique du racisme, t. I. L'Empire du démenti, Paris, La Découverte, 2021.

25. Voir Jacques Derrida, "Quand j'ai entendu l'expression "délit d'hospitalité"... », intervention publique prononcée le 21 décembre 1996 au théâtre des Amandiers (Nanterre) [URL : http://www.gisti.org/spip. php?article3736].

26. Judith Butler, Qu'est-ce qu'une vie bonne?, Martin Rueff (trad. fr. et préface), Paris, Payot, 2014.

27. Joan Stavo-Debauge, "Venir à la communauté : une sociologie de l'hospitalité et de l'appartenance ", thèse de doctorat de sociologie sous la direction de Laurent Thévenot, Paris, EHESS, 2009.

28. François Jullien, Les Transformations silencieuses, Paris, Le Livre de poche, 2010, p. 28.

29. Voir Marion Howa, "Augmenter l'architecture", dans Hutin, 2021, cité n. 2, p. 130-133.

30. Voir David Edgerton, "Techniques créoles et histoires mondiales. Repenser le déplacement des objets dans l'espace et dans le temps », Emmanuel Grimaud, Denis Vidal, Yann-Philippe Tastevin (dir.), Low tech? Wild tech!, numéro thématique de Techniques et Culture, $n^{\circ} 67,2017$, p. 30-63, ici p. 48.

31. « $[\mathrm{A}]$ growing number of people are increasingly called to use their "natural" designing abilities; because the sentence "we are all designers" no longer refers to a potential, but to a reality with we must face up to » (Ezio Manzini, Design, When Everybody Designs: An Introduction to Design for Social Innovation, Cambridge, The MIT Press, 2015, p. 30, traduction de l'auteure).

32. Baptiste Morizot, Manières d'être vivant : enquête sur la vie à travers nous, Arles, Actes Sud, 2020 ; Diplomates. Cohabiter avec les loups sur une autre carte du vivant, Marseille, Wildproject, 2016.

33. Empruntée à Eduardo Viveiros de Castro.

34. Voir Claude Lévi-Strauss, La Pensée sauvage, Paris, Plon, 1962.

35. Michel de Certeau, Luce Giard, Pierre Mayol, L'Invention du quotidien, t. II. Habiter, cuisiner, Paris, Gallimard, 1994, p. 51.

36. Cette image a été tout particulièrement explorée dans Colin Rowe, Fred Koetter, Collage city (1978), Kenneth Hylton (trad. fr.), Gollion, Infolio, 2002.

37. Qui fait l'objet de ma thèse de doctorat d'architecture, "La Conception par bricolage comme art de la situation. Architecture, urbanisme, design ", École nationale supérieure d'architecture de Toulouse / université Toulouse - Jean-Jaurès, 2020.

38. Citons, pour les plus connus, Steward Brand, Whole Earth Catalog: Access to Tools, New York, Point / Random House, 1968 et Bernard Rudofsky, Architecture Without Architects. An Introduction to Non-Pedigreed Architecture, cat. exp. (New York, The Museum of Modern Art, 1964-1965), New York, The Museum of Modern Art, 1964.

39. Jo Freeman, "The Tyranny of Structurelessness" (1971), Berkeley Journal of Sociology, vol. 17, 19721973, p. 151-165 [URL : www.jofreeman.com/joreen/ tyranny.htm].

40. Christopher Dell, Christophe Hutin, « Être tout à fait libre !", dans Hutin, 2021, cité n. 2, p. 70-100, ici p. 71.

41. "Working constructively with contingency in an assemblage of actors, things, actions, and discourses on site ». Christopher Dell, The Improvisation of Space, Berlin, Jovis, 2019, p. 6.

42. Ibidem, et Christopher Dell, La Ville comme partition ouverte, Zürich, Lars Müller, 2016.

43. Ibid.

44. Voir notamment les travaux du groupe Pièces et main d'œuvre, "site de bricolage pour la construction d'un esprit critique grenoblois » [URL : http://www.piecesetmaindoeuvre.com/spip.php?page=plan]

45. Voir Bruno Latour (dir.), Reset Modernity!, cat. exp. (Karlsruhe, Zentrum für Kunst und Medien, 16 avril 21 août 2016) Cambridge, MIT Press, 2016.

46. Voir Matthieu Duperrex, «Le pipeline et la clôture de la frontière ", dans Pierre-Henry Frangne et al. (dir.), Des lignes et des paysages. Du sillon à la skyline, Rennes, Presses universitaires de Rennes, 2020, p. 159-179.

47. Voir Jan Zalasiewicz et al., "Scale and Diversity of the Physical Technosphere: A Geological Perspective", The Anthropocene Review, vol. 4, n 1, 2017.

48. Voir Hélène Tordjman, La Croissance verte contre la nature, Paris, La Découverte, 2021. 
49. Voir Matthieu Duperrex, " D'une passe à poissons. Milieux et technique en Anthropocène ", Techniques et Culture, 2019 [URL : http://journals.openedition. org/tc/10920].

50. Voir Matthieu Duperrex, Mikaëla Le Meur (dir.), Semer le trouble. Soulèvements, subversions, refuges, numéro thématique de Techniques et Culture, $\mathrm{n}^{\circ} 74$, 2020.

51. Voir Anna Lowenhaupt Tsing, "Vers une théorie de la non-scalabilité ", Multitudes, vol. 82, n 1, 2021, p. 65-71.

52. Saskia Sassen, Expulsions. Brutalité et complexité dans l'économie globale, Gallimard, Paris, 2016, p. 296. 